\title{
LETTERS
}

\section{Candida auris can be identified accurately}

I read with interest the recent CMAJ article by Schwartz and Dingle, which gives a good synopsis of Candida auris. ${ }^{1}$ However, the authors' claim that certain matrixassisted laser desorption/ionization time of flight (MALDI-TOF) mass spectrometry identification systems can misidentify C. auris is somewhat misleading.

The article by Tsay and colleagues ${ }^{2}$ cited by Schwartz and Dingle states that accurate identification for $C$. auris can be performed by VITEK MS and Bruker Biotyper MALDI-TOF mass spectrometry. Public Health Ontario also states that these 2 methods can identify C. auris. ${ }^{3}$ Furthermore, a recent study of 50 molecularly confirmed $C$. auris isolates from different geographical areas showed 100\% accurate identification by MALDI-TOF mass spectrometry. ${ }^{4}$ Despite the small sample, that is more than the 19 cases of C. auris identified in Canada since 2012. ${ }^{1}$

I would like to gently caution that Schwartz and Dingle's misleading claim could lead to clinicians and laboratorians unnecessarily questioning the identification of Candida species by MALDI-TOF mass spectrometry, and consequently overtesting and misusing resources. Patients may also be misled, which is not helpful in establishing good physician-patient rapport.

\section{Eugene Y.H. Yeung MD MSc}

Resident physician, Department of Medical Microbiology, University of Ottawa; Eastern Ontario Regional Laboratory Association, Ottawa, Ont.

- Cite as: CMAJ 2020 January 13;192:E44. doi: $10.1503 / \mathrm{cmaj} .73957$

\section{References}

1. Schwartz IS, Dingle TC. Candida auris. CMAJ 2019;191:E865.

2. Tsay S, Kallen A, Jackson BR, et al. Approach to the investigation and management of patients with Candida auris, an emerging multidrug-resistant yeast. Clin Infect Dis 2018;66:306-11.

3. Candida auris reference identification and susceptibility testing. Toronto: Public Health Ontario; 2019. Available: www.publichealthontario.ca/-/media/ documents/lab/lab-sd-131-candida-auris-reference -id-susceptibility-testing.pdf?la=en (accessed 2019 Oct. 18).

4. Vatanshenassan M, Boekhout T, Meis JF, et al. Candida auris identification and rapid antifungal susceptibility testing against echinocandins by MALDI-TOF MS. Front Cell Infect Microbiol 2019;9:20.

Competing interests: None declared. 